(C) 2011 IEEE. Personal use of this material is permitted. Permission from IEEE must be obtained for all other uses, in any current or future media, including reprinting/republishing this material for advertising or promotional purposes, creating new collective works, for resale or redistribution to servers or lists, or reuse of any copyrighted component of this work in other works 


\section{Optimization Design of a Voice Coil Actuator Based on Improved SOM}

\author{
Y. B. Li \\ School of Electronic Information \\ Zhongyuan University of Technology \\ Zhengzhou, China \\ liyanbin@189.cn
}

\author{
G. Lei, Y. G. Guo, and J. G. Zhu \\ Faculty of Engineering and Information Technology \\ University of Technology, Sydney \\ Sydney, Australia \\ Gang.Lei@uts.edu.au
}

\begin{abstract}
Voice coil actuator is a new kind of direct drive motor. It has many good performances, such as high acceleration and fast response. We present an improved sequential optimization method (SOM) and dimension reduction optimization method to design optimization a cylindrical voice coil actuator in this work. In the implementation, design of experiments (DOE) technique and Kriging approximate model are employed to improve the optimization efficiency. From the discussion, we can see that the proposed methods are very efficient. And computation cost of finite element analysis can be reduced remarkably (more than $2 / 3$ of the cost has been saved) by the proposed methods.
\end{abstract}

Keywords - Design of Experimants, finite element analysis, Kriging model, sequrntial optimization method, voice coil actuator.

\section{INTRODUCTION}

Voice coil actuator is a kind of device which can convert the electric energy into mechanical energy, and realize specified rectilinear or limited oscillation angle motions. Generally, two kinds of voice coil actuators have been widely investigated namely linear and rotated voice coil actuators. In this work, we consider the design optimization problem of a cylindrical voice coil actuator (CVCA).

About the optimization of motor/actuator, there are many optimization methods/algorithms. For the algorithms, a lot of algorithms have been developed, such as simulated annealing algorithm (SA), genetic algorithm (GA), tabu search (TS), clonal selection algorithm (CSA) and differential evolution algorithm (DEA) [1].

For the optimization models, three kinds of models have been widely employed. First is the finite element model. This is a physical model for the real model. It is accurate, but it always leads to a large amount of computation cost, especially for the three dimensional electromagnetic design optimization problems. Second is the magnetic equivalent circuit model. Third is the approximate model, such as the response surface model, radial basis function model and Kriging model and artificial neural networks model. In these models, response surface model and radial basis functions model are the deterministic parameter model [2]-[5]. Kriging model is a semi-parameter model [6], [7]. Artificial neural networks model is a non-parameter model [8], [9]. They have been widely used in the engineering optimization design problems. Each model has its advantages and disadvantages. And several comparison works about the models' optimization efficiency have been investigated [10].

In traditional methods, algorithms and models are always discussed separately. However, from the point of view of engineering optimization, they should be optimized simultaneously. Therefore, in our previous work, we present a sequential optimization method (SOM) for the low dimensional optimization problems. And dimension reduction optimization method (DROM) has been presented for the higher dimensional optimization problems [11]-[12]. From the investigated two TEAM workshop problems, TEAM problem 22 and 25, we can see that the proposed methods are very efficient to those problems. So in this work, we present the improved methods for the optimization design of a CVCA. The following section is an introduction for the CVCA. The third Section is an introduction of Kriging model. The fourth section is the presentation of the improved SOM and dimension reduction optimization method, followed by the discussions and results.

\section{CylindRicAl Voice COIL ACTUATOR}

Fig. 1 illustrates an axisymmetrical model of the studied CVCA [13], [14]. It includes a PM (material is NdFeB), two nonlinear cores and a moving coil. Fig. 2 shows the design parameters and several fixed parameters. There are seven design parameters for this problem, which are illustrated in the Table I, including their ranges.

TABLE I

THE DESIGN PARAMETERS AND RANGES

\begin{tabular}{|c|l|c|c|c|}
\hline$x_{1}$ & height of PM & unit & $\min$ & $\max$ \\
\hline$x_{2}$ & width of PM & $\mathrm{mm}$ & 8.25 & 30 \\
\hline$x_{3}$ & height of coil & $\mathrm{mm}$ & 1 & 30 \\
\hline$x_{4}$ & width of coil & $\mathrm{mm}$ & 9.25 & 59.75 \\
\hline$x_{5}$ & height of core below the PM & $\mathrm{mm}$ & 0.35 & 30 \\
\hline$x_{6}$ & height of core up the PM & $\mathrm{mm}$ & 1 & 30 \\
\hline$x_{7}$ & width of core & $\mathrm{mm}$ & 1 & 30 \\
\hline
\end{tabular}

For the optimization, one objective and five constraints are defined in this work. The objective is the total mass of the 
device. The first constraint considers the coil mass, which should be equal to $10 \mathrm{~g}$. The second constraint is about the force, which should be equal to $5 \mathrm{~N}$ when the parameter $\mathrm{D}$ is $4.25 \mathrm{~mm}$. The third constraint is about the mean magnetic field $B$ in the area of 1, 2 and 3 illustrated in the Fig. 3. The last two constraints are about the geometry dimension. Then the optimization model is defined as follows.

Min : $f=$ Mass of total device

$$
\text { s.t. }\left\{\begin{array}{l}
\mathrm{g}_{1}: \text { Coil mass }=10 \mathrm{~g} \\
\mathrm{~g}_{2}: F=5 N, \text { when } D=4.25 \mathrm{~mm} \\
\mathrm{~g}_{3}: \overline{\mathrm{B}}_{i} \leq 1 \mathrm{~T} \text { in area } 1,2,3 \text { in Fig.3, } \\
\mathrm{g}_{4}: s_{\min }+s_{\max }+x_{6} \leq x_{3} \\
\mathrm{~g}_{5}: x_{3} \leq x_{1}+x_{6}-s_{\min }
\end{array}\right.
$$

where $s_{\min }$ is $0.25 \mathrm{~mm}, s_{\max }$ is $8.25 \mathrm{~mm}$, which are fixed parameters. Furthermore, $p_{1}$ and $p_{2}$ in Fig. 2 are $0.9 \mathrm{~mm}$ and $0.725 \mathrm{~mm}$, respectively. Fig. 4 shows the B-H curve of the material of core.

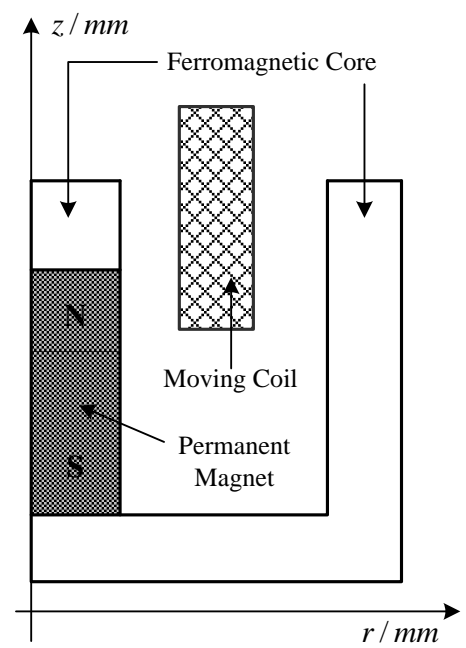

Fig. 1. The material design model of CVCA

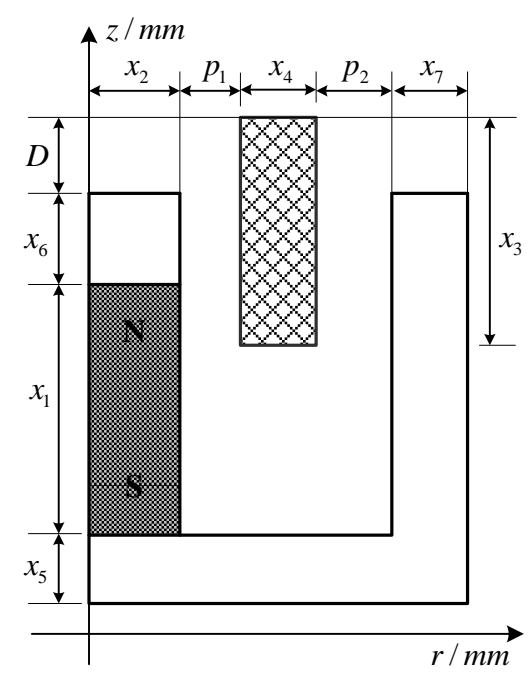

Fig. 2. The design parameters of CVCA

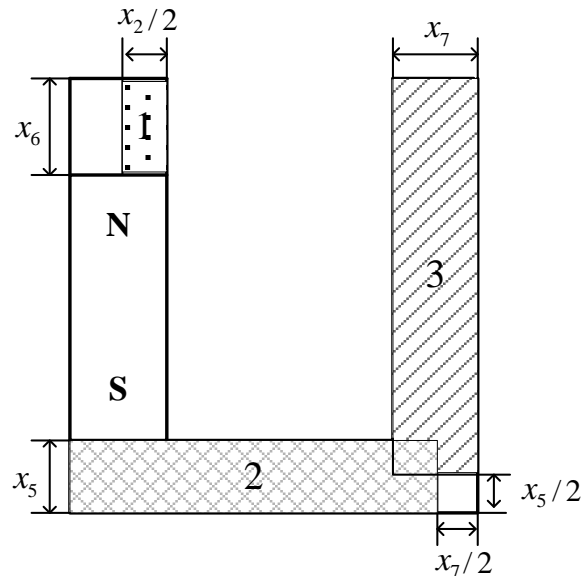

Fig. 3. The magnetic relevant part in the third constraint

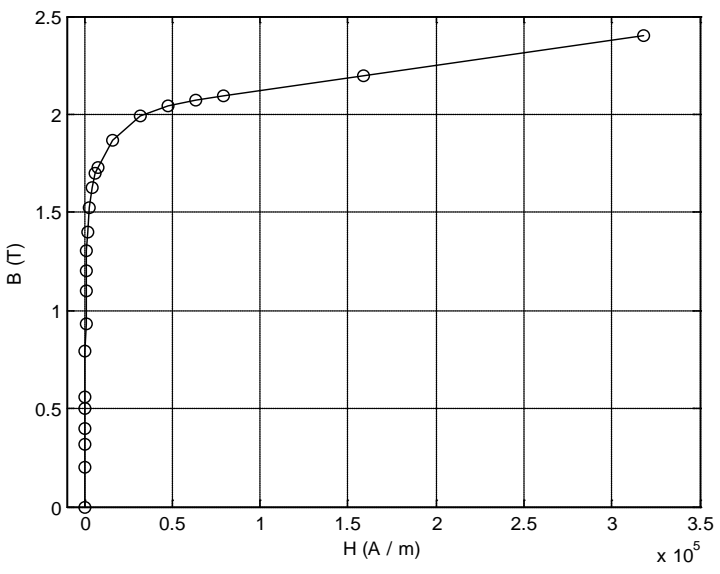

Fig. 4. B-H curve of the core material

\section{KRIGING MODEL}

Kriging model is composed of a determined model and a stochastic model, which has the forms as

$$
\hat{y}(x)=f(x) \boldsymbol{\beta}+z(x),
$$

where $f(x)$ is the determined model, such as response surface model, $\boldsymbol{\beta}$ is the model parameter vector, $z(x)$ is non-zero covariance matrix $\mathbf{C}=\left[c_{i j}\right]$ with zero mean and variance $\sigma^{2}$ [6], [7], [10], [15]. It has the form as

$$
c_{i j}=\sigma^{2} \mathbf{R}\left[r\left(\mathbf{x}_{i}, \mathbf{x}_{j}\right)\right],
$$

where $\mathbf{R}$ is the correlation matrix, and $r\left(\mathbf{x}_{i}, \mathbf{x}_{j}\right)$ is the userspecified correlation function.

$$
\mathbf{R}=\left[\begin{array}{cccc}
r\left(\mathbf{x}_{1}, \mathbf{x}_{1}\right) & r\left(\mathbf{x}_{1}, \mathbf{x}_{2}\right) & \cdots & r\left(\mathbf{x}_{1}, \mathbf{x}_{n}\right) \\
r\left(\mathbf{x}_{2}, \mathbf{x}_{1}\right) & r\left(\mathbf{x}_{2}, \mathbf{x}_{2}\right) & \cdots & r\left(\mathbf{x}_{2}, \mathbf{x}_{n}\right) \\
\vdots & \vdots & \ddots & \vdots \\
r\left(\mathbf{x}_{n}, \mathbf{x}_{1}\right) & r\left(\mathbf{x}_{n}, \mathbf{x}_{2}\right) & \cdots & r\left(\mathbf{x}_{n}, \mathbf{x}_{n}\right)
\end{array}\right]
$$


Gaussian correlation functions are most commonly used. They have the form as

$$
r\left(x_{i}, x_{j}\right)=\exp \left\{-\sum_{k=1}^{n} \alpha_{k}\left|x_{i}-x_{j}\right|^{2}\right\}
$$

To construct a Kriging model, there are three parameters $\beta$ in (2), $\sigma^{2}$ in (3) and $\alpha_{k}$ in (5) must be estimated. There are many methods to obtain those parameters, such as minimum mean square error estimation method and maximum likelihood method. And fortunately, all parameters can be easily estimated by the software package DACE (Design and Analysis of Computer Experiments) [7].

\section{DROM AND IMPROVED SOM}

Fig. 5 illustrates the optimization flowchart of the proposed method, the combination of dimension reduction optimization and improved sequential optimization method. It mainly contains the following steps.

1) Define the problem, including the objective, constraints. Generally, the problem defined in this step is a higher dimensional problem.

2) Sample some initial points with an algorithm or a design of experiments (DOE) technique. With the sampled points, we can use some analysis method to determine the significant factors. Generally, two methods can be used, namely analysis of variance (ANOVA) technique or grey correlation analysis (GCA) technique. ANOVA is a classic factor-significance analysis method based on the DOE. GCA technique is a sensitivity and significance analysis method which is conducted to evaluate the importance of the design factors. The basic concept of GCA is to determine if a relationship among a series of data is close, based on the degree of similarity among the geometric shapes of the data series curves. In the implementation, maybe there are some impractical points for ANOVA. In this case, we will use Kriging model to construct the approximate model for the studied parameter/response firstly, then we can get the responses from this approximate model and implement the analysis process.

3) From the former step, we can get the significant factors and non-significant factors. Then we select the three or four highly significant factors as a new optimization problem. Then the initial higher dimensional design optimization problem can be converted into a lower dimensional problem.

4) Optimize the lower dimensional design optimization problem with the improved SOM [12]. There are two optimization processes in SOM: coarse optimization process (COP) and fine optimization process (FOP). The main purpose of the former is to reduce the design space; while the target of the latter is to update the optimal design parameters.

In the implementation, Kriging model will be used in the step 2 and step 4 to reduce the computation cost of finite element analysis. Detailed SOM parameters, such as the space reduction factor in the COP and the sample updating method, can be seen in the previous work.

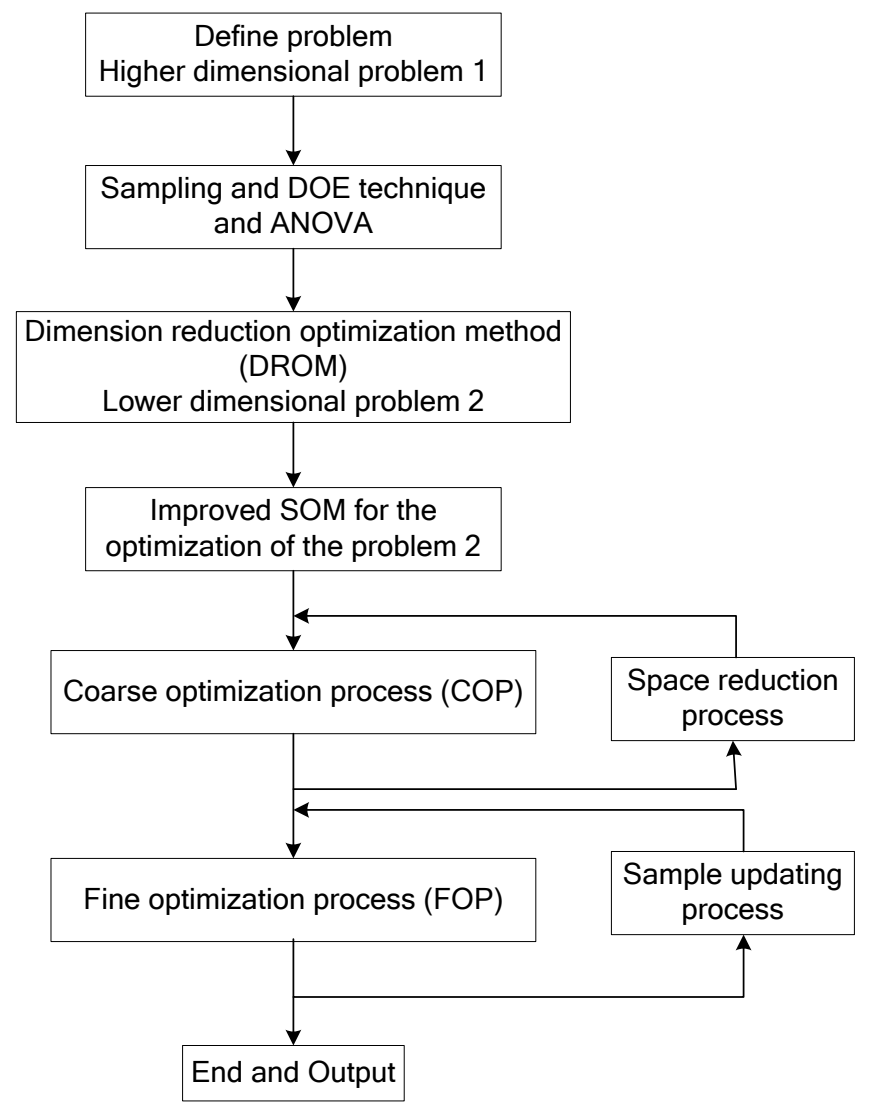

Fig. 5. The flowchart of DROM and improved SOM

\section{DISCUSSIONS AND RESULTS}

Differential evolution algorithm (DEA) was used in this work as the optimization algorithm. DEA is a relatively new evolutionary optimization algorithm [1].

In the basic DEA, the initial population of each generation is sampled from uniform distribution. It should be noted that the design space has been updated in each optimization process in improve SOM, so the initial population in DEA must be changed corresponding. In the implementation, the algorithm parameters are selected as follows: population size is 35 , mutation scaling factor is 0.8 , crossover factor is 0.8 , and the maximum number of iteration is 1000 .

Table II lists the optimization solutions for CVCA with the proposed method. Two solutions are shown in the table.

1) For the direct optimization with finite element model and DEA (DEA column), the obtained optimal design parameters are [9.8682, 8.9773, 13.4858, 1.7226, 3.1425, $4.4563,2.5688$ ]; total mass is $79.20 \mathrm{~g}$. The obtained force is $5.01 \mathrm{~N}$, the mass of the coil is $9.94 \mathrm{~g}$. And to get this solution, 6725 finite element sample points (FESP) are needed.

2) For the optimization with the proposed method, two strategies are illustrated in the table. One is that 1200 points are sampled in the DROM process, and the corresponding results are listed in the DROM-1 column. The other is that 2000 points are sampled in the DROM process, and the corresponding results are listed in the DROM-2 column. 


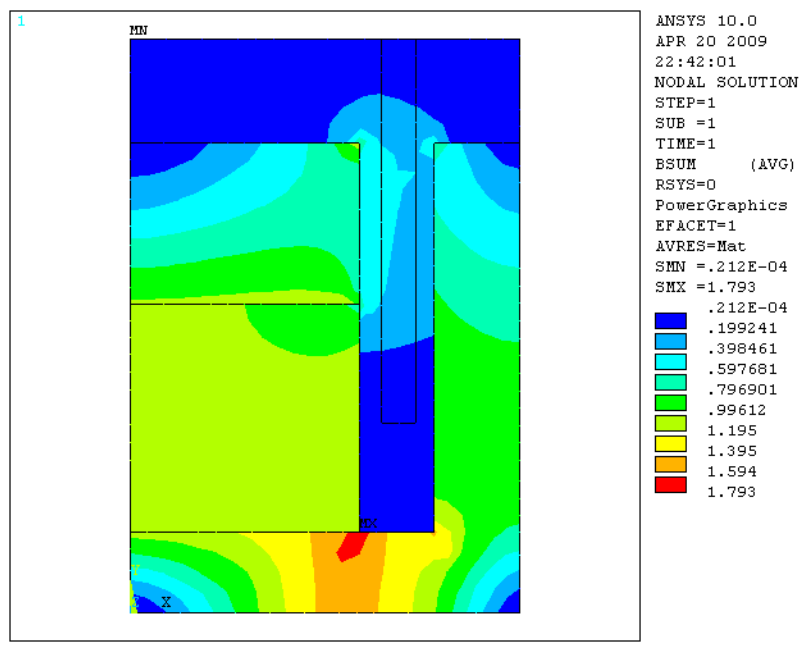

Fig. 5. The magnetic filed from the design scheme of DROM-2

TABLE II

OPTIMIZATION RESULTS OF CVCA

\begin{tabular}{|c|c|c|c|c|}
\hline Par. & unit & DEA & DROM-1 & DROM-2 \\
\hline$x_{1}$ & $\mathrm{~mm}$ & 9.8682 & 13.7305 & 9.4132 \\
\hline$x_{2}$ & $\mathrm{~mm}$ & 8.9773 & 9.2540 & 9.4661 \\
\hline$x_{3}$ & $\mathrm{~mm}$ & 13.4858 & 15.1734 & 15.8112 \\
\hline$x_{4}$ & $\mathrm{~mm}$ & 1.7226 & 1.5156 & 1.4321 \\
\hline$x_{5}$ & $\mathrm{~mm}$ & 3.1425 & 14.5104 & 3.3249 \\
\hline$x_{6}$ & $\mathrm{~mm}$ & 4.4563 & 6.1880 & 6.6480 \\
\hline$x_{7}$ & $\mathrm{~mm}$ & 2.5688 & 2.1692 & 3.5696 \\
\hline Force & $\mathrm{N}$ & 5.01 & 5.20 & 5.12 \\
\hline Mass coil & $\mathrm{g}$ & 9.94 & 10.00 & 10.00 \\
\hline Mass total & $\mathrm{g}$ & 79.20 & 155.18 & 106.08 \\
\hline FESP & - & 6725 & 1395 & 2044 \\
\hline
\end{tabular}

For the case of DROM-1, the obtained total mass is 155.18g, which is bigger that from direct optimization method (DEA). However, the constraint of mass coil (which is equal to $10 \mathrm{~g}$ ) is better than that from DEA. And the needed FESP is only 1395 , which is only about $20.74 \%$ compared with that of DEA. So the computation cost has been reduced a lot.

On the other hand, if we select 2000 points in the DROM, then from the optimization, we can get the optimal design parameters are [9.4132, 9.4661, 15.8112, 1.4321, 3.3249, $6.6480,3.5696]$. And the gained total mass is $106.08 \mathrm{~g}$, which is smaller than the case of DROM-1. Meanwhile, the needed FESP is only 2044, which is only about $30 \%$ compared with that of DEA.

\section{CONSLUSION}

In this work, we present a combination method of the improved SOM and DROM to deal with the optimization design problem of CVCA. From the comparison, we can see more than $2 / 3$ of the computation cost of finite element analysis can be reduced by the presented method. And the obtained solution is also very satisfactory. Therefore, the proposed method is very efficient to deal with the optimization design problem of electromagnetic devices.

\section{ACKNOWLEDGMENT}

This work was supported by the Natural Science Foundation of Department of Education of Henan Province under grant 2011A470015.

\section{REFERENCES}

[1] R. Storn and K. Price, "Differential evolution-A simple and efficient heuristic for global optimization over continuous spaces," Journal of Global Optimization, vol. 11, pp. 341-359, 1997.

[2] M. Dorica and D. D. Giannacopoulos, "Response clustering for electromagnetic modeling and optimization," IEEE Transactions on Magnetics,vol. 42, no. 4, pp. 1127-1130, 2006.

[3] J. L.Coulomb, A. Kobetski, M. C. Costa et al. Comparison of radial basis function approximation techniques. The International Journal for Computation and Mathematics in Electrical and Electronic Engineering (COMPEL), vol.22, no. 3, pp. 616-629, 2003.

[4] H. M. Gutmann, “A radial basis function method for global optimization,” Journal of global optimization, vol.19, no. 3, pp. 201-227, 2001.

[5] T. Ishikawa, Y. Tsukui, and M. Matsunami, "A combined method for the global optimization using radial basis function and deterministic approach," IEEE Transactions on Magnetics, vol. 35, no. 3, pp. 1730-1733, 1999.

[6] L. Lebensztajn, C. A. R. Marretto, M. C. Costa, and J. L. Coulomb, "Kriging: a useful tool for electromagnetic device optimization," IEEE Transactions on Magnetics, vol. 40, no. 2, pp. 1196-1199, 2004.

[7] S. N. Lophaven, H. B. Nielsen, and J. Sondergaard, "DACE: A MATLAB Kriging toolbox version 2.0,” Technical Report IMM-TR-2002-12, Technical University of Denmark, Copenhagen, 2002.

[8] I. Marinova, C. Panchev, and D. Katsakos, "A neural network inversion approach to electromagnetic device design,” IEEE Transactions on Magnetics.,vol. 36, no. 4, pp. 1080-1084, July 2000.

[9] P. Burrascano, S. Fiori, and M. Mongiardo, "Review of artificial neural networks applications in microwave computer-aided design," Int. J. RF Microw. CAE, vol. 9, no. 3, pp. 158-174, 1999.

[10] L. D. Wang and D. A Lowther, "Selection of approximation models for electromagnetic device optimization,” IEEE Transactions on Magnetics., vol. 42, no. 2, pp. 1227-1230, 2006.

[11] Gang Lei, K. R. Shao, Youguang Guo, Jianguo Zhu, and J. D. Lavers, "Improved sequential optimization method for high dimensional electromagnetic optimization problems," IEEE Transactions on Magnetics,, vol. 45, no. 10, pp. 3993-3996, 2009.

[12] Gang Lei, K. R. Shao, Youguang Guo, Jianguo Zhu, and J. D. Lavers, "Sequential design of experiments techniques for the optimization design of electromagnetic devices,” in Proc. $14^{\text {th }}$ CEFC, May 9-12, 2010, Chicago, USA. DOI: 10.1109/CEFC.2010.5481481.

[13] L. Encica, J. Makarovic, E. A. Lomonova, A. J. A. Vandenput, "Space mapping optimization of a cylindrical voice coil actuator,” IEEE Trans. on Ind. Appl., vol. 42, no. 6, pp. 1437-1444, 2006.

[14] D. Echeverría1, D. Lahaye1, L. Encica, E. A. Lomonova, P. W. Hemker, A. J. A. Vandenput, "Manifold-mapping optimization applied to linear actuator design,” IEEE Trans. Magn., vol. 42, no. 4, pp.1183-1186, 2006.

[15] Y. B. Li, J. H. Chen, and G. Lei, "Model Selection Strategy for the Sequential Optimization Design of Electromagnetic Devices," in Proc. 2010 ICEMS, pp. 1826-1829, 2010. 